oxidative stress model. SR $\mathrm{Ca}^{2+}$ release was measured by treating cells loaded with fluorescent dye, fluo-4-AM, with caffeine. Cardioprotection was tested by exposing ARVC to metabolic ischaemiareperfusion. Ned-19 was found to significantly delay the time to mPTP opening by $76 \% \pm 16 \%, 55 \% \pm 20 \%, 47 \% \pm 19 \%$ and $44 \% \pm 17 \%$ (all $\mathrm{p}<0.05$ ) at concentrations of $100 \mu \mathrm{mol} / 1,10 \mu \mathrm{mol} / \mathrm{l}, 1 \mu \mathrm{mol} / \mathrm{l}$ and $0.1 \mu \mathrm{mol} / 1$, respectively, compared with the control group. Concentrations of Ned-19 at $100 \mu \mathrm{mol} / 1,10 \mu \mathrm{mol} / 1$ and $1 \mu \mathrm{mol} / \mathrm{l}$, but not $0.1 \mu \mathrm{mol} / 1$, significantly inhibited caffeine-stimulated SR $\mathrm{Ca}^{2+}$ release $(71.6 \% \pm 2.0 \%, 34.2 \% \pm 1.9 \%, 55.6 \% \pm 5.5 \%$ and $-14 \% \pm$ $21 \%$, respectively) indicating non-specific effects at higher concentrations. A low dose of $0.1 \mu \mathrm{mol} / \mathrm{l}$ Ned-19 increased the survival of cells following metabolic ischaemia-reperfusion to $46 \% \pm 19 \%$ from $29 \%$ (control).

In conclusion, we have shown the involvement of NAADP in SR $\mathrm{Ca}^{2+}$ release and mPTP opening, and that by inhibiting NAADP signalling at reperfusion with Ned-19, cardiomyocytes may be protected against ischaemia-reperfusion injury.

\section{BAS/ CARDIOPROTECTION BY HYPOXIA-INDUCIBLE FACTOR-1 $\alpha$ : BSCR33 UNDERLYING BENEFICIAL EFFECTS ON MITOCHONDRIAL FUNCTION}

doi:10.1136/hrt.2010.205781.44

${ }^{1} S-G$ Ong, ${ }^{1} S$ Y Lim, ' $\mathrm{L}$ Theodorou, ${ }^{2} \mathrm{D}$ H Shukla, ${ }^{2} \mathrm{P}$ H Maxwell, 'D M Yellon, ${ }^{1} \mathrm{D} J$ Hausenloy. ${ }^{1} T h e$ Hatter Cardiovascular Institute, University College London Hospital \& Medical School, UK; ${ }^{2}$ Centre for Cell Signalling and Molecular Genetics, University College London, UK

Introduction Activation of hypoxia-inducible factor- $1 \alpha$ (HIF-1 $\alpha$ ) protects the heart from ischaemia-reperfusion injury, although the underlying mechanisms are unclear. We hypothesised that HIF-1 $\alpha-$ induced cardioprotection is mediated by beneficial effects on mitochondrial function.

Methods and results Two different experimental models of HIF- $1 \alpha$ activation were used: (1) pharmacological inhibition of proline hydroxylase (PHD) and (2) genetic inactivation of von Hippel-Lindau (VHL), proteins responsible for HIF-1 $\alpha$ degradation under normoxic conditions. A single dose $(3 \mathrm{mg} / \mathrm{kg})$ of the PHD inhibitor (GSK0360A or PHDi), administered by oral gavage $4 \mathrm{~h}$ before ex vivo myocardial infarction, reduced myocardial infarct size (percentage of the area at risk) in male Sprague-Dawley rats $(30.6 \% \pm 2.9 \%$ PHDi vs $44.2 \% \pm 2.9 \% ; \mathrm{p}<0.5 ; \mathrm{N}>5)$. Next, conditional cardiac-specific VHL knockout mice (VHL-KO) that express an inducible Cre-recombinase transgene to delete the VHL-floxed gene within the heart following tamoxifen induction, expressed higher levels of HIF-1 in the heart as assessed by immunostaining. The activation of myocardial HIF-1 resulted in a smaller myocardial infarct size in comparison with the littermate control $(29.1 \% \pm 4.7 \%$ in VHL-KO vs $52.5 \% \pm 3.3 \%$ in control; $\mathrm{p}<0.05 ; \mathrm{N}>5$ /group). In VHL-KO cardiomyocytes subjected to simulated ischaemiareperfusion injury (SIRI) (120 min ischaemia and $15 \mathrm{~min}$ reperfusion, the production of reactive oxygen species (ROS) (measured by reduced Mitotracker Red fluorescence) $(1.0 \% \pm 0.1$-fold increase in VHL-KO vs $1.3 \% \pm 0.2$-fold increase in control; $\mathrm{p}<0.05 ; \mathrm{N}>3$ experiments each with 40 cells) and mitochondrial permeability transition pore (mPTP) opening sensitivity was reduced (measured by TMRM fluorescence) $(1.1 \% \pm 0.1$ fold increase in VHL-KO vs $1.4 \% \pm 0.1$ fold increase in control; $\mathrm{p}<0.05 ; \mathrm{N}>3$ experiments each with 40 cells).

Conclusions HIF- $1 \alpha$ activation by genetic deletion of VHL or pharmacological inhibition of $\mathrm{PHD}$, is cardioprotective and this protective effect can be attributed in part to beneficial effects on the mitochondria.

\section{BAS/ BSCR34 IS AN INCREASED AMPK ACTIVATION DURING ISCHAEMIA ESSENTIAL FOR THE PROTECTION OF THE HEART AGAINST INFARCTION?}

doi:10.1136/hrt.2010.205781.45

${ }^{1,2} \mathrm{M}$ Paiva, ${ }^{2} \mathrm{~L}$ M Goncalves, ${ }^{2} \mathrm{~L}$ A Providencia, ${ }^{1} \mathrm{~S} M$ Davidson, ${ }^{1} \mathrm{M} M \mathrm{M}$ Mocanu, ${ }^{1} \mathrm{D}$ M Yellon. ${ }^{1}$ The Hatter Cardiovascular Institute, University College London, UK; ${ }^{2}$ Basic Research in Cardiology Unit, Cardiology Department, Coimbra University Hospital and Coimbra Medical School, Coimbra, Portugal

Background During ischaemia, AMPK activation occurs in order to provide energy from alternative resources. However, AMPK activity is known to be impaired in diabetes. We hypothesised that enhancing AMPK activation above physiological levels during ischaemia would protect both the normoglycaemic and the diabetic heart.

Methodology Hearts from Wistar and Goto Kakizaki rats (GK, a mildly diabetic rat strain) were subjected to 35 min coronary artery occlusion in the presence of 10, 20 or $40 \mu \mathrm{M}$ A-769662 (an activator of AMPK), followed by $120 \mathrm{~min}$ of reperfusion with normal buffer $(\mathrm{n} \geq 6)$. Risk zone and myocardial infarction were assessed using Evans blue and 2,3,5-triphenyltetrazolium chloride (TTC) staining, respectively and expressed as percentage of the area at risk (I/R\%). The effect of A-769662 on mitochondrial permeability transition (opening of the mPT pore is associated with reperfusion injury) was also investigated by exposing rat cardiomyocytes loaded with the fluorophore TMRM to a laser oxidative insult; the time to mitochondrial membrane depolarisation and rigour contracture were measured ( $n=6,80-100$ cells/assay).

Results A-769662 reduced the infarct size in both the normoglycaemic and diabetic hearts in comparison with control hearts at 20 $\mu \mathrm{M}(31.8 \% \pm 3.1$ vs $51.4 \% \pm 1.5$ normoglycaemic heart; $22.7 \% \pm 3.0$ vs $37.6 \% \pm 2.7$ for the GK heart; $\mathrm{p}<0.05)$ and at $40 \mu \mathrm{M}(35.6 \% \pm 1.9$ vs $51.4 \% \pm 1.5$ for the normoglycaemic heart; $18.6 \% \pm 1.6$ vs $37.6 \% \pm 2.7$, for the GK heart; $\mathrm{p}<0.05$ ) In addition, A-769662 also significantly delayed the mPTP opening $(147.71 \% \pm 10.2 \%$ at $20 \mu \mathrm{M}, 146.7 \% \pm$ $15.6 \%$ at $40 \mu \mathrm{M}$, vs control $100 \%, \mathrm{p}<0.05)$.

Conclusions Our data suggest that the enhancement of AMPK activity during ischaemia may lead to infarct reduction and delayed opening of the MPTP in the ischaemic reperfused rat heart.

\section{BAS/ EFFECTS OF ALDOSTERONE AND OBESITY ON THE BSCR35 ANTICONTRACTILE PROPERTIES OF PERIVASCULAR ADIPOSE TISSUE IN RAT AORTIC RINGS}

doi:10.1136/hrt.2010.205781.46

Fiona M Lynch, Abbigail Howson, Sarah B Withers, Anthony M Heagerty. Cardiovascular Research group, School of Biomedicine, University of Manchester, Core Technology Facility, Manchester, UK

The mechanisms by which perivascular adipose tissue (PVAT) can reduce vascular contractility remain to be elucidated and may underlie the associations of obesity with hypertension, insulin resistance and cardiovascular disease. This study investigates the effects of aldosterone and obesity in isolated rat aorta. Healthy and obese male rats were killed by stunning and cervical dislocation. The mesenteric bed was removed and arteries dissected with and without PVAT. Arteries were mounted on a wire myograph and were constricted with $60 \mathrm{mM}$ KPSS. Cumulative concentration responses $\left(10^{-9}-10^{-5} \mathrm{M}\right)$ to norepinephrine (NE) were performed before and after $10 \mathrm{~min}$ incubation with aldosterone $(5 \mathrm{nM})$. Endothelial integrity was confirmed by relaxation to $10^{-5} \mathrm{M}$ acetylcholine. Responses are expressed as mean ( \pm SEM) percentage of KPSS constriction and analysed using two-way ANOVA. PVAT $(n=10)$ significantly $(p<0.05)$ reduced constriction in healthy vessels 
(PVAT: 81 (20)\% vs no PVAT: 140 (27)\% at $10^{-5} \mathrm{M} \mathrm{NE}$ ). The anticontractile properties of PVAT are abolished $(n=6)$ in the presence of aldosterone (PVAT: 53 (24)\% vs no PVAT: 62 (35)\% at $10^{-5} \mathrm{M} \mathrm{NE}$ ). Aldosterone significantly reduced tension $(p<0.05)$ compared with no PVAT controls. The anticontractile properties of PVAT $(n=6)$ are absent in arteries from obese animals (PVAT: 105 (33)\% vs no PVAT: $91(15) \%$ at $10^{-5} \mathrm{M} \mathrm{NE}$ ); however, in the presence of aldosterone $(n=6)$ constrictions are significantly increased $(\mathrm{p}<0.05)$ in arteries with (PVAT: 133 (49)\% vs no PVAT: $89(18) \%$ at $10^{-5} \mathrm{M} \mathrm{NE}$ ). These results demonstrate that obesity and aldosterone impair the anticontractile effects of PVAT. Aldosterone reduces contractility in healthy arteries but increases contractility in obese arteries.

\section{BAS/ REGULATION OF HUMAN SMOOTH MUSCLE CELL BSCR36 DEVELOPIMENT BY MYOCARDIN}

doi:10.1136/hrt.2010.205781.47

L Raphel, S Sinha. Division of Cardiovascular Medicine, University of Cambridge, UK

Background Myocardin is a cardiac-specific and smooth muscle cell (SMC)-specific transcription factor with a key role in development. It regulates a wide variety of SMC-specific contractile markers by acting as an accessory protein for serum response factor, binding to its DNA binding sites (CArG boxes), and so has a major effect on SMC phenotype. However, its precise role in SMC development remains unclear. Moreover, there are no data on its requirement in human SMC development.

Methodology We investigated whether myocardin was required for SMC development from human embryonic stem cells in an embryoid body model and whether we could promote SMC development at high efficiency by overexpressing myocardin.

Results \& conclusions Embryoid bodies from human embryonic stem cells were found to express increasing quantities of SMC markers up to 60 days of development with the appearance of visibly contractile SMC patches as a late phenomenon. Overexpression of myocardin using an adenovirus vector increased differentiation of pluripotent cells into the SMC lineage, although only a subset of cells was susceptible to the overexpressed transcription factor. Loss of function studies using a truncated myocardin dominant negative construct resulted in only minor or no reduction in SMC differentiation, suggesting that redundant pathways exist during embryonic development in human cells.

\section{BAS/ DISCOVERY AND CHARACTERISATION OF NOVEL PEPTIDE BSCR37 AGONISTS AND THE FIRST ANTAGONIST FOR THE CARDIOVASCULAR PEPTIDES, THE APELINS}

\section{doi:10.1136/hrt.2010.205781.48}

${ }^{1} \mathrm{~S} L$ Pitkin, ${ }^{2} \mathrm{~N} J \mathrm{M}$ Macaluso, ${ }^{1} \mathrm{~J} \mathrm{~J}$ Maguire, ${ }^{2} \mathrm{R}$ C Glen, ${ }^{1} \mathrm{~A}$ P Davenport. ${ }^{1}$ Clinical Pharmacology Unit, University of Cambridge, Level 6 Centre for Clinical Investigation, Addenbrooke's Hospital, Cambridge, UK; ${ }^{2}$ Unilever Centre for Molecular Sciences Informatics, Department of Chemistry, University of Cambridge, Cambridge, UK

The recently discovered apelin family of peptides mediate their actions by a single G-protein coupled receptor, APJ. We have previously shown that apelins have three major actions in the human cardiovascular system: endothelium-dependent vasodilatation; direct vasoconstriction by interacting with smooth muscle APJ receptor and increased cardiac contractility by action on cardiac myocytes. Our aim was to discover shorter sequences of (Pyr1)apelin-13 retaining agonist activity and use a computational ligand-based strategy to design cyclic peptide agonists and antagonists. Over 50 compounds were synthesised and tested in a competition binding assay. Functional assays for agonists measured vasoconstrictor action in endothelium-denuded human saphenous veins. Antagonist activity was measured in cyclic AMP assays against (Pyr1)apelin-13. Data are expressed as mean \pm SE, $\mathrm{pD} 2=-\log 10 \mathrm{EC} 50, \quad \mathrm{Emax}=$ maximum response. The predicted cleavage product of the angiotensin converting enzyme 2 (ACE-2), apelin-13(1-12), inhibited radiolabelled apelin binding and was identified as the shortest sequence potently constricting endothelium-denuded saphenous vein (pD2 9.07\% \pm 0.40 , Emax $29.30 \% \pm 9.43 \% \mathrm{KCl}, \mathrm{n}=5$ ). The most potent cyclic analogue identified, MM07, inhibited binding with a $\mathrm{KD}=86 \% \pm 30 \mathrm{nM}$, $(n=3)$ and $\mathrm{pD} 210.53 \% \pm 0.24$, Emax $21.80 \% \pm 5.72 \% \mathrm{KCl}, \mathrm{n}=3)$ with a comparable potency and efficacy to (Pyr1)apelin-13 (pD2=8.8\% $\pm 0.3, \quad$ Emax $26 \% \pm 4 \%, n=15) . \quad$ MM54 inhibited binding, $\mathrm{KD}=3.42 \% \pm 0.45 \mu \mathrm{mol} / \mathrm{l},(\mathrm{n}=3)$ and was identified as an antagonist with a pA2 value of 5.9 in a cyclase inhibition assay. The novel cyclic peptide MM07 retains potency and we have identified the first apelin receptor antagonist, MM54 as a pharmacological tool to characterise the apelin system and in the design of small molecule drugs.

Acknowledgements We thank the BHF for support.

\section{BAS/ CYTOKINE PROFILING IN CULTURE REVEALS A BSCR38 PREDOMINANCE OF M1 MACROPHAGE POLARISATION IN SYMPTOMATIC CAROTID PLAOUES}

doi:10.1136/hrt.2010.205781.49

${ }^{1} \mathrm{~J}$ Shalhoub, ${ }^{2} \mathrm{~A}$ Cross, ${ }^{1} \mathrm{D}$ Allin, ${ }^{2} \mathrm{D}$ Essex, ${ }^{1} \mathrm{~A}$ H Davies, ${ }^{2} \mathrm{C}$ Monaco. ${ }^{1}$ Imperial Vascular Unit, Imperial College London, Charing Cross Hospital London, UK; ${ }^{2}$ Cytokine Biology of Atherosclerosis Group, Kennedy Institute of Rheumatology, Imperial College, London, UK

Rationale Macrophages in atherosclerotic plaques are a heterogeneous population. To uncover signatures of classical (M1) or alternative (M2) macrophage polarisation during plaque instability, we compared cytokine and chemokine production via Luminex profiling in asymptomatic human carotid plaques with carotid plaques in the territory of recent focal neurological symptomatology.

Methodology Carotid endarterectomy specimens were collected from 50 consenting patients ( 26 symptomatic, 24 asymptomatic). Fresh specimens were divided symmetrically along their long axis, allowing for representative undertaking of both immunohistochemistry and plaque cell culture (Monaco et al. PNAS USA 2004;101:5634-9). Automated image analysis (Clemex Vision) quantified CD68 staining. Cells were isolated via enzymatic dissociation to produce a viable mixed cell suspension and cultured for $24 \mathrm{~h}$. Macrophage was the predominant cell type in the mixed cell culture. Supernatants were interrogated with a panel of 22 cytokines and chemokines on a Luminex 100 platform.

Results CD68 immunopositivity was significantly higher in symptomatic than in asymptomatic plaques $(p=0.0075)$. Luminex detected 17 of the 22 analytes, with a predominance of myeloidderived cytokines over lymphoid-derived cytokines, in keeping with the predominance of macrophage in culture. Tumour necrosis factor $\alpha$, interleukin (IL)-1 $\alpha$, IL- $1 \beta$, IL- 6 , granulocyte-macrophage colonystimulating factor, CCL2, CCL5 and IL-10 levels were significantly higher in symptomatic plaques.

Conclusions Our data demonstrate that symptomatic atherosclerotic carotid disease is associated with a cytokine pattern consistent with the predominance of proinflammatory M1-type macrophage polarisation. M2-dependent cytokine IL-10 was also part of this inflammatory response, suggesting macrophage heterogeneity. Our study has implications for future therapeutic and diagnostic applications for high-risk atherosclerosis. 